\title{
REVISTA
}

\section{DIMENSIONAMENTO AMOSTRAL PARA FRUTOS EM CULTIVARES COMERCIAIS DE MAMOEIRO AVALIADOS A CAMPO}

\section{SAMPLE SIZE FOR FRUITS IN COMMERCIAL PAPAYA CULTIVARS EVALUATED IN THE FIELD}

\author{
Jeferson Pereira Ferreira ${ }^{1}$ \\ Omar Schmildt ${ }^{2}$ \\ Clemilton Alves da Silva ${ }^{3}$ \\ Adriel Lima Nascimento ${ }^{4}$ \\ Edilson Romais Schmildt ${ }^{5}$ \\ Rodrigo Sobreira Alexandre ${ }^{6}$ \\ Laercio Francisco Cattaneo ${ }^{7}$ \\ Geraldo Antônio Ferreguetti ${ }^{8}$ \\ Vinicius de Souza Oliveira ${ }^{9 *}$
}

\footnotetext{
${ }^{1}$ Universidade Federal do Espírito Santo, CEUNES, ES, Brasil; jeferson.p.ferreira@hotmail.com ${ }^{2}$ Universidade Federal do Espírito Santo, CEUNES, ES, Brasil; omarschmildt@gmail.com ${ }^{3}$ Universidade Federal do Espírito Santo, CEUNES, ES, Brasil; clemiltonallves@gmail.com ${ }^{4}$ Universidade Federal do Espírito Santo, CEUNES, ES, Brasil; adriel_aln@outlook.com

${ }^{5}$ Universidade Federal do Espírito Santo, CEUNES, ES, Brasil; e.romais.s@gmail.com ${ }^{6}$ Universidade Federal do Espírito Santo, DCFM, ES, Brasil; rodrigosobreiraalexandre@gmail.com ${ }^{7}$ Faculdades Integradas Espírito-Santenses (FAESA), ES, Brasil; lfcattaneo@hotmail.com ${ }^{8}$ Caliman Agrícola S/A, Linhares, Espírito Santo, Brasil; geraldo@caliman.com.br ${ }^{9}$ Universidade Federal do Espírito Santo, CCAE, ES, Brasil; souzaoliveiravini@gmail.com *Autor para correspondência
}

Artigo submetido em 19/05/2020, aceito em 19/08/2020 e publicado em 28/12/2020.

Resumo: O objetivo deste trabalho foi estimar o tamanho de amostra (número de frutos) necessário para avaliar os caracteres, massa de frutos (g) e teor de sólidos solúvéis ( ${ }^{\circ}$ Brix), em cultivares comerciais de mamoeiro THB, Golden, Tainung e UENF/Caliman 01, por meio da técnica bootstrap de simulação por reamostragens. Para as simulações foram utilizados 75 frutos de cada cultivar estudado. Os resultados encontrados indicaram tamanhos amostra para avaliação experimental de frutos das cultivares UENF/Caliman 01, Golden, Tainung 01 e THB são de 29, 37, 45 e 48 frutos respectivamente, com erro de estimação de $5 \%$ da média.

Palavras-chave: Carica papaya L., reamostragem, bootstrap. 
Abstract: The objective of this work was to estimate the sample size (number of fruits) necessary to evaluate the characters, fruit mass (g) and soluble solids content ( $\left.{ }^{\circ} \mathrm{Brix}\right)$, in the commercial papaya plantations of THB, Golden, Tainung and UENF/Caliman 01, by the means of the bootstrap technique for the simulation of re-sampling. For the simulation, 75 fruits were utilized from each studied plantation. The results found indicate sample sizes for experimental evaluation of fruits of the cultivars UENF / Caliman 01, Golden, Tainung 01 and THB with 29, 37, 45 and 48 fruits, respectively, with an error of $5 \%$ of the average.

Keywords: Carica papaya L., resampling, bootstrap.

\section{INTRODUÇÃO}

O Brasil é destaque na produção mundial de mamão, sendo o segundo maior produtor, com cerca de 1.060.392 toneladas de frutos no ano de 2018, contribuindo com 8\% de toda produção mundial (FAO, 2020). O estado do, Espírito Santo se destaca com produtividade de 354.405 toneladas, representam mais de $33 \%$ da produção nacional (IBGE, 2020). Além da importância econômica da cultura, também têm o aspecto social, gerando emprego e renda, absorvendo mão de obra durante o ano todo, pela necessidade constante de manejo, tratos culturais, colheita e comercialização efetuadas de maneira contínua nas lavouras, além da renovação de plantios que ocorre em média a cada três anos (BENASSI, 2007)

Dada a importância da cultura do mamoeiro para o Brasil no cenário mundial, são desenvolvidas muitas pesquisas com essa cultura à nível de campo. A qualidade da análise desses dados experimentais depende do correto dimensionamento da amostra. O tamanho da amostra é influenciado por vários fatores, dentre os quais, destacam-se a variabilidade inerente à característica e a precisão amostral definida pelo pesquisador.

Com relação ao nível de precisão, é notório que quanto menor for o erro estabelecido pelo pesquisador para estimação de parâmetros, maior será a amostra exigida para estimativas precisas (STORCK et al., 2011). A determinação do tamanho amostral é fundamental em qualquer experimento científico, pois se o tamanho da amostra for reduzido, pode diminuir a precisão experimental (HELL et al., 2017) o que pode até invalidar o trabalho (COELHO et al., 2011), por outro lado, se as amostras forem excessivamente grandes, exigem dispêndio de recursos financeiros, tempo e mão de obra, dada assim a importância de mensurar amostras que representam a população (BURIN et al., 2014).

Na literatura são encontrados trabalhos que tratam de tamanho amostral para características de frutos de algumas frutíferas como Citrus sinensis (AVANZA et al., 2010), Litchi chinensis (ANDRADE e JASPER, 2012), Prunus persica (TOEBE et al., 2012), Ananas comosus (KRAUSE et al., 2013), Malus domestica (TOEBE et al., 2014a), Carya illinoinensis (CARGNELUTTI FILHO et al., 2015; POLETTO et al., 2018), Passiflora spp. (BANDEIRA et al., 2016; SCHMILDT et al., 2017) e, Spondias purpurea (SILVA et al., 2016). Concernente ao Carica papaya L., têm-se trabalho em ambiente packing house (SILVA et al., 2017b) e em cultivo protegido (SCHMILDT et al., 2019), entretanto, nenhum trabalho de dimensionamento amostral foi encontrado para caracteres relacionados a frutos de mamoeiro em condições de campo.

Diante da importância da cultura do mamoeiro e ao dimensionamento amostral, objetivou-se com esse trabalho determinar o tamanho amostral mínimo necessário para a caracterização da massa de frutos (g) e do teor de sólidos solúveis ( ${ }^{\circ}$ Brix), utilizando a técnica estatística bootstrap de reamostragem (EFRON, 1979). 


\section{MATERIAL E MÉTODOS}

O experimento foi conduzido na fazenda Caliman Agrícola S/A, localizada em Linhares, Espírito Santo (Latitude de $19^{\circ}$ $11^{\prime} 49^{\prime \prime} \mathrm{S}$ e Longitude de $40^{\circ} 05^{\prime} 52^{\prime \prime} \mathrm{O}$ ).

Foram selecionados aleatoriamente 300 frutos de mamoeiro no estágio de maturação 1/4, em lavouras comercias no início do ciclo produtivo. Sendo que desses 300 frutos, 75 frutos pertenciam a cada um dos cultivares estudados (THB, Golden, Tainung 01 e UENF/Caliman 01).

Os pomares dos cutivares THB, Tainung 01 e UENF/Caliman 01 foram implantados no verão, enquanto o cultivar UENF/Caliman 01 foi implantado no outono. Os tratos culturais foram realizados seguindo as necessidades eventuais da cultura, e o espaçamento entre plantas foi de $3,60 \times 1,50 \mathrm{~m}$ para os cultivares THB e Golden, e 3,60 x 2,0 m para os cultivares Tainung 01 e UENF/Caliman 01. Os frutos foram colhidos no verão do ano de 2014, todos no mesmo dia.

Em cada fruto foram mensurados os seguintes caracteres: massa de frutos (MFR) - medido em balança de precisão, expresso em gramas considerando duas casas decimais e considerando apenas frutos comerciais; teor de sólidos solúveis (SS) determinados, em frutos 1/4 maduros, por leitura direta em refratômetro de bancada, e expresso em ${ }^{\circ}$ Brix.

Para caracterizar o conjunto de dados a partir das avaliações dos 75 frutos de cada cultivar, procedeu-se a análise por meio da estatística descritiva. Para caracterizar as possíveis diferenças entre as médias de cada caractere entre os quatro cultivares realizou-se a comparação pelo teste de Student-Newman-Keuls.

Para determinar o tamanho de amostra utilizou-se o procedimento com amostragens com reposição via bootstrap. Para as simulações, foram planejados 75 tamanhos de amostra para cada caractere de cada cultivar de mamoeiro, sendo o tamanho de amostra inicial de um fruto, e os demais obtidos com incrementos de um até atingir 75 frutos. Portanto, para as simulações, foram planejados os seguintes tamanhos de amostra: 1, 2, 3, ..., 75 frutos para cada caractere de cada cultivar.

A seguir, para cada tamanho de amostra planejado de cada caractere de cada cultivar, foram realizadas 4.000 simulações, por meio de reamostragem, com reposição. Para cada amostra simulada, foi estimada a média. Assim, para cada tamanho de amostra de cada caractere de cada cultivar, foram obtidas 4.000 estimativas da média (FERREIRA, 2009). Depois, calculou-se a amplitude do intervalo de confiança de $95 \%$ (IC 95\%) pela diferença entre o percentil 97,5\% e o percentil 2,5\% (CARGNELUTTI FILHO et al., 2012) representando-se graficamente.

A seguir, determinou-se o tamanho de amostra (número de frutos) para a estimação da média de cada caractere de cada cultivar pela análise gráfica. Para essa determinação, partiu-se do tamanho inicial (um fruto) e considerou-se como tamanho de amostra o número de plantas a partir do qual as médias se mantiveram dentro do limite do intervalo de confiança à 95\%.

As análises estatísticas foram realizadas com o auxilio do programa R (R CORE TEAM, 2019) e do Office Excel (LEVINE et al., 2012).

\section{RESULTADOS E DISCUSSÃO}

Os resultados da estatística descritiva para a massa de frutos e teor de sólidos solúveis são apresentados na Tabela 1. Verifica-se que a média para massa de frutos dos cultivares do grupo "Formosa" (Tainung 01 e UENF/Caliman 01) foi superior à média encontrada dos cultivares do grupo "Solo" (THB e Golden), o que era de se esperar pelo fato dos dois grupos heteróticos "Formosa" e "Solo" se diferirem principalmente em relação à massa de frutos (DANTAS et al., 2002; CARDOSO et al., 2014), o que corrobora com os resultados encontradas por Silva et al. (2017a) ao 
estudarem a divergência genética em 59 acessos de mamoeiro, tanto do grupo "Solo" quanto do "Formosa", evidenciando que a massa de fruto é a característica que mais contribuiu para a diversidade genética, usando o método de Singh (1981), baseado no D2 de Mahanalobis, que considera a característica de maior importância, a de maior variabilidade.

Os resultados de massa encontrados neste trabalho estão em conformidade com padrão de frutos que atenda ao mercado nacional, ao qual exige frutos com massa entre 800 a $1500 \mathrm{~g}$, e ao mercado externo, que exige massa em torno de $500 \mathrm{~g}$ (DIAS et al., 2011). Outros autores também obtiveram frutos com este padrão de mercado, tanto nos genótipos do grupo 'Solo', com média de 390 g para o Golden (DIAS et al., 2011) e, 438,76 g em THB (NASCIMENTO et al., 2018a), quanto aos genótipos do grupo 'Formosa', com 1049, 75 g para o UENF/ Caliman 01 (NASCIMENTO et al., 2018b) e, 963,85 g em Tainung 01 (SILVA et al., 2018).

Tabela 1. Estatística descritiva com valores mínimo, máximo, média, coeficiente de variação

(CV) e teste de normalidade de Lilliefors (L) para os caracteres massa de frutos e teor de sólidos solúveis em frutos de mamoeiro (Carica papaya L.) cultivar THB, Golden, Tainung 01 e UENF/Caliman 01

\begin{tabular}{cccrccc}
\hline Cultivar & Mínimo & Máximo & Média $^{(1)}$ & $\mathrm{CV}(\%)$ & $\mathrm{L}^{(2)}$ \\
\hline \multicolumn{7}{c}{ Massa de frutos (grama por fruto) } \\
\hline THB & 312,60 & 771,50 & 547,82 & c & 17,64 & $\mathrm{~S}$ \\
Golden & 316,10 & 758,60 & 522,67 & c & 15,50 & $\mathrm{~S}$ \\
Tainung 01 & 721,50 & 2023,50 & $1372,08 \quad$ a & 16,76 & $\mathrm{~S}$ \\
UENF/Caliman 01 & 740,00 & 1514,00 & $1121,66 \quad$ b & 13,79 & $\mathrm{~S}$ \\
\hline \multicolumn{7}{c}{ Teor de sólidos solúveis ( ${ }^{\circ}$ Brix) } \\
\hline THB & 8,00 & 14,00 & $11,23 \quad$ c & 8,02 & $\mathrm{~N}$ \\
Golden & 10,00 & 13,75 & $12,38 \quad$ b & 5,45 & $\mathrm{~S}$ \\
Tainung 01 & 9,00 & 13,50 & 11,12 & c & 8,99 & $\mathrm{~S}$ \\
UENF/Caliman 01 & 10,00 & 16,00 & 13,08 a & 8,94 & $\mathrm{~N}$ \\
\hline
\end{tabular}

(1) Médias seguidas pela mesma letra na comparação entre os cultivares para cada caractere, não diferem estatisticamente entre si pelo teste de Student-Newman-Keuls à 5\% de probabilidade de erro.

(2) $\mathrm{S}=$ distribuição normal; $\mathrm{N}=$ distribuição não - normal.

O teor médio de sólidos solúveis (SS), em ${ }^{\circ}$ Brix, foram maiores para a cultivar UENF/Caliman 01 pertencente ao grupo "Formosa” (Tabela 1). Outros autores também encontraram valores médios de SS similares a este experimento, tanto no grupo "Formosa", como Rodolfo et al. (2007), Barros et al. (2017), e Nascimento et al. (2018b) com variações de 11,2 a 14,6 ${ }^{\circ}$ Brix, quanto no grupo "Solo", como Fagundes e Yamanishi (2001), Silva et al. (2017b), e Nascimento et al. (2018a) obtendo valores com variações de 9,9 a 12,5 ${ }^{\circ}$ Brix. Esta diferenciação no teor de SS acontece porque durante a fase de maturação dos frutos ocorre um aumento no teor de açúcares, o que é variável de acordo com o tipo de mamão, cultivar, condições climáticas, fertilidade do solo, época de produção, e estágio de desenvolvimento de maturação (FAGUNDES e YAMANISHI, 2001), contudo, nota-se na Tabela 1 que as médias de SS das cultivares, estão dentro da faixa definida na Instrução Normativa 4/2010 de Padrão de Identidade e Qualidade (PIQ) do Ministério da Agricultura e Pecuária (MAPA, 2010), que estabelece valores mínimos de $11^{\circ}$ Brix.

No que concerne à distribuição dos dados, apenas os cultivares THB e 
UENF/Caliman 01 apresentaram um distanciamento da normalidade, para o teor de sólidos solúveis. Entretanto, o bootstrap pode ser usado tanto na estatística paramétrica quanto na não paramétrica. Então, diante dessas considerações, em relação à normalidade, pode-se inferir que os dados referentes a esses caracteres oferecem credibilidade ao estudo do dimensionamento de amostra.

O bootstrap paramétrico consiste em gerar reamostras baseadas na distribuição de probabilidades conhecidas, utilizando como parâmetros dessa distribuição a estimativa das mesmas, obtida na amostra mestre (FERREIRA, 2009). É usado quando o interesse está em estimar o vício, teste de hipóteses das estimativas dos parâmetros, e principalmente estabelecer o intervalo de confiança de determinada distribuição de dados (NAVIDI, 2006).

A maior variabilidade dos dados é observada no caractere massa de frutos, sendo maior no cultivar THB $(\mathrm{CV}=17$, 64\%), seguido pelo cultivar Tainung 01 (CV $=16,76 \%$ ). Comumente, o tamanho da amostra está relacionado diretamente à variabilidade dos dados, representado pelo coeficiente de variação; e inversamente proporcional ao erro de estimação (amplitudes do intervalo de confiança) permitido da característica estudada
(BARBETTA et al., 2004). Isso acontece porque um aumento do tamanho da amostra reduz a variância da média amostral desde que a variância amostral permaneça constante (LAW e KELTON, 2000).

Assim, quanto maior o tamanho da amostra, maior a precisão e, consequentemente a diminuição do coeficiente de variação amostral, o que permite inferir que quando o erro experimental que é a variação entre as parcelas é significativamente superior ao erro amostral que é a variação dentro da parcela, o número de repetições do experimento ou número de indivíduos por parcela (tamanho de amostra) deve ser aumentado (STORCK et al., 2011).

Na Figura 1 observam-se os valores mínimo, máximo, as médias e os percentis da variável massa de frutos, em cada tamanho amostral simulado com base em 4000 estimativas. O mesmo tipo de gráfico é apresentado para a característica teor de sólidos solúveis na Figura 2. Percebe-se que as médias dos frutos por amostra simulada para os diferentes cultivares de mamoeiro estão todos dentro do IC 95\%. Isso indica que esses 75 frutos são suficientes para a determinação do tamanho amostral dos caracteres dos cultivares estudados, por meio da técnica de reamostragem utilizada. 
Figura 1. Valores de média, mínimo, percentil 2,5\%, percentil 97,5\% e máximo das 4.000 médias da massa de frutos, obtidas por reamostragens, em cada tamanho de amostra (número de frutos). Reamostragens realizadas com base na massa de 75 frutos de quatro cultivares de mamoeiro (Carica papaya L.).
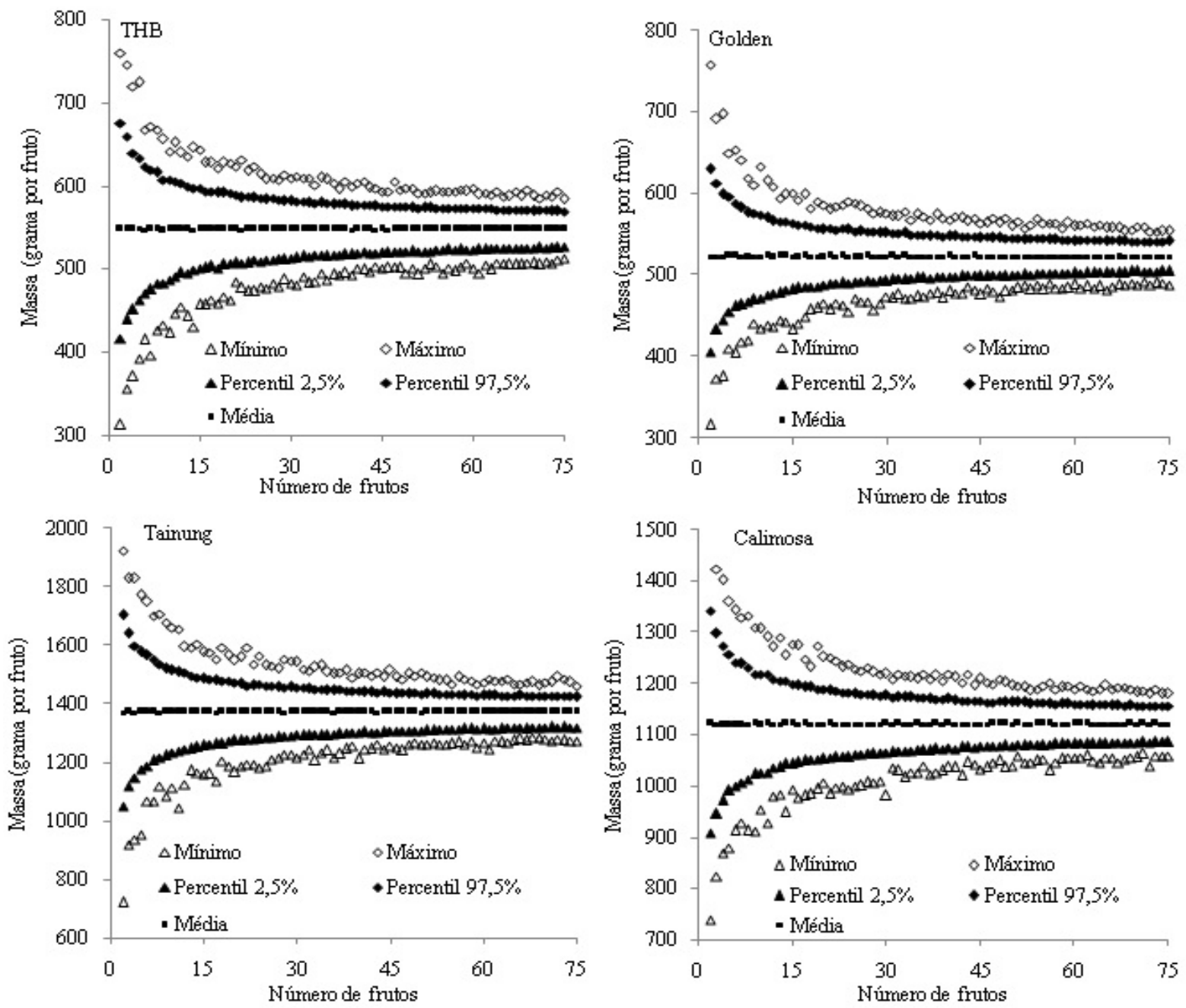
Figura 2. Valores de média, mínimo, percentil 2,5\%, percentil 97,5\% e máximo das 4.000 médias do teor de sólidos solúveis, obtidas por reamostragens, em cada tamanho de amostra (número de frutos). Reamostragens realizadas com base no teor de sólidos solúveis de 75 frutos de quatro cultivares de mamoeiro (Carica papaya L.).
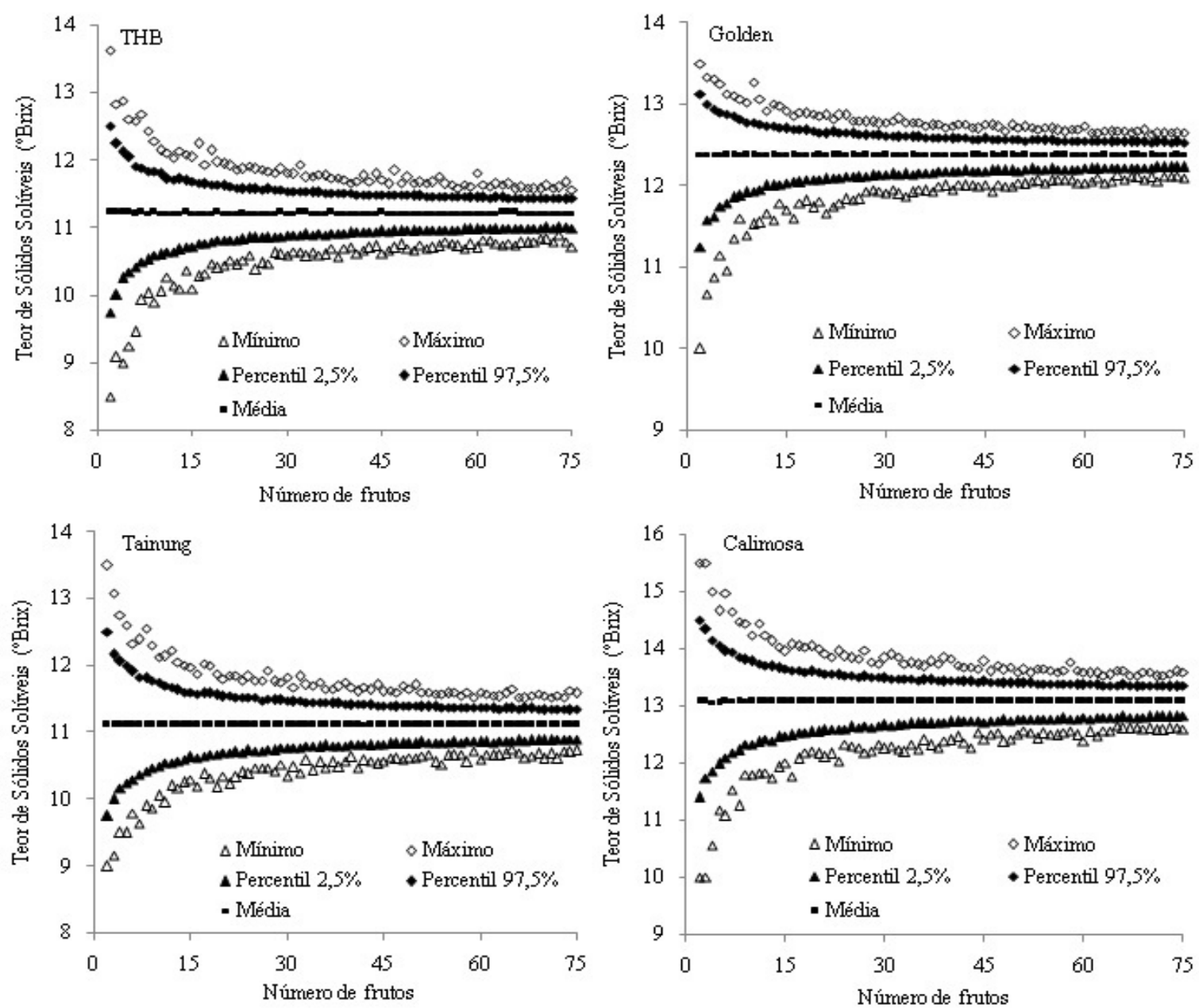

Os tamanhos de amostra (número de frutos) para a estimação da média de massa de frutos para uma amplitude do intervalo de confiança de bootstrap de $95 \%$ a $5 \%$ de erro, variaram desde 29 até 48 frutos, respectivamente, para os cultivares UENF/Caliman 01 e THB (Tabela 2). O tamanho da amostra foi menor para o teor de sólidos solúveis (Tabela 2). Portanto,

para o caractere teor de sólidos solúveis do cultivar Golden, por exemplo, amostras com seis frutos são suficientes para a determinação desta característica com a mesma precisão das amostras iniciais de 75 frutos, com uma margem de erro inferior a $5 \%$. Isso implica em uma redução de $92 \%$ dos gastos para avaliação desta característica. 
Tabela 2. Estimativas do número mínimo de frutos de mamoeiro (Carica papaya L.) por amostra simulada para os caracteres massa de frutos e teor de sólidos solúveis, para os cultivares THB, Golden, Tainung 01 e UENF/Caliman 01

\begin{tabular}{cccc}
\hline \multirow{2}{*}{ Cultivar } & \multicolumn{3}{c}{ Amplitudes do intervalo de confiança de 95\% } \\
\cline { 2 - 4 } & $5 \%$ & Massa $\left(\right.$ g.fruto $\left.{ }^{-1}\right)$ & $15 \%$ \\
\hline THB & 48 & 12 & 6 \\
Golden & 37 & 9 & 4 \\
Tainung 01 & 45 & 11 & 5 \\
UENF/Caliman 01 & 29 & 7 & 3 \\
\hline & Teor de sólidos solúveis $\left({ }^{\circ}\right.$ Brix) \\
\hline THB & 11 & 3 & 2 \\
Golden & 2 & 2 \\
Tainung 01 & 13 & 3 & 2 \\
UENF/Caliman 01 & 12 & 3 & \\
\hline
\end{tabular}

Em termos práticos o número elevado de frutos para o caractere massa de frutos, não é um fator limitante para o dimensionamento amostral, uma vez que essa característica é de fácil mensuração e de análise não destrutiva, o que possibilita a reutilização dos frutos para outras analises ou até mesmo ao consumo.

Em determinação de tamanho de amostra para frutos de mamoeiro THB, após tratamento pós-colheita em packing house, Silva et al. (2017b) também encontraram maior tamanho de amostra para a característica massa de frutos em relação à característica teor de sólidos solúveis. Os referidos autores afirmam que, com erro de 5\% em torno da média, 33 frutos é o tamanho ótimo para avaliar a característica massa de frutos, valor este inferior aos 48 frutos encontrados neste trabalho. Esta diferença pode ser entendida pelo fato de os frutos avaliados neste trabalho, não terem sofrido nenhuma seleção como é feita após a chegada no packing house.

Concernente à estimação do tamanho de amostra, outros autores, trabalhando com outras culturas, também detectaram a necessidade de maior tamanho de amostra para avaliação de massa de fruto em relação ao teor de sólidos solúveis, e dentre estes destacamos a avaliação em frutos de pêssego por Toebe et al. (2012) e de maracujá por Schmildt et al. (2017).

$\mathrm{Na}$ prática, os resultados desse trabalho, possibilitam ao pesquisador escolher o tamanho de amostra, para estimar a média desses caracteres com adequada precisão (Tabela 2). Por exemplo, para estimar a média com a maior precisão (5\% da estimativa da média) em frutos de THB, 48 frutos seriam suficientes para avaliação experimental de massa de teor de sólidos solúveis.

Assim, ao planejar um experimento para ser conduzido com frutos de THB, recém-colhidos, em delineamento experimental inteiramente casualizado, para a estimação da média de cada tratamento com 5\% de precisão, devem ser avaliadas 48 frutos por tratamento. Se o experimento for planejado com quatro repetições por tratamento, seriam amostrados 12 frutos por repetição $(48 / 4=12)$, ou seja, 12 frutos por parcela. Ainda, se no experimento fossem avaliados cinco tratamentos seriam amostrados 240 frutos (48 por tratamento).

\section{CONCLUSÃO}

O tamanho de amostra requerido para avaliar massa de frutos é maior do que para avaliar teor de sólidos solúveis nas 
cultivares THB, Golden, Tainung 01 e UENF/Caliman 01.

Para avaliação experimental de frutos das cultivares THB, Golden, Tainung 01 e UENF/Caliman 01, são requeridos tamanho de amostra de 48, 37, 45 e 29 frutos respectivamente, com intervalo de confiança de bootstrap de $95 \%$ com erro de estimação de $5 \%$ da média.

\section{AGRADECIMENTOS}

A CAPES, FAPES e ao CNPq (processo 311181/2018-0) pelas bolsas concedidas aos autores e a Caliman Agrícola S/A pela logística na realização do experimento.

\section{REFERÊNCIAS}

ANDRADE, R.A.; JASPER, S.P. Unidade amostral para determinação de massa média de frutos em lichieira em sistema orgânico e convencional. Comunicata Scientiae, v.3, n.2, p.139-142, 2012.

AVANZA, M.M.; BRAMARDI, S. J.; MAZZA, S. M. Tamaño óptimo de muestra para evaluar el patrón de crecimiento de frutos de naranjo 'Valencia Late'. Revista Brasileira de Fruticultura, v.32, n. 4, p.1154-1163, 2010.

BANDEIRA, C.T.; GIORDANA, S. K.; TOEBE, M.; SAIFERT, L.; GIOCOBBO, C. L.; WELTER, L. J. Sample size for estimate the average of Passiflora caerulea fruits traits. Ciência Rural, v.46, n.10, p.1729-1736, 2016.

BARBETTA, P.A.; REIS, M. M.; BORNIA, A. C. Estatística para cursos de engenharia e informática. $4^{\mathrm{a}}$ ed. São Paulo: Atlas, 2014, 410p.

BARROS, F.L.S.; KUHLCAMP, K. T.; ARANTES, S. D.; MOREIRA, S. O. Productivity and quality of Formosa and
Solo papaya over two harvest seasons. Pesquisa Agropecuária Brasileira, v.52, n.8, p.599-606, 2017.

BENASSI, A.C. 2007. Informes sobre a produção do mamão. São Paulo: Toda Fruta. Disponível em: <http://www.todafruta.com.br/todafruta/mo stra_conteudo.asp?conteudo=14291>.

Acesso em: 14 set. 2017.

BURIN, C.; CARGENELUTTI FILHO, A.; TOEBE, M.; ALVES, B. M.; FICK, A. L. Dimensionamento amostral para a estimação da média e da mediana de caracteres de tremoço branco (Lupinus albus L.). Comunicata Scientiae, v.5, n.2, p.205-212, 2014.

CARDOSO, D.L.; LUZ, L. N.; MACÊDO, C. M. P.; GONÇALVES, L. S. A.; PEREIRA, M. G. Heterosis in papaya: inter and intra group analysis. Revista Brasileira de Fruticultura, v.36, n.3, p.610- 619, 2014

CARGNELUTTI FILHO, A.; POLETTO, T. MUNIZ, M. F. B.; BAGGIOTTO, C.; POLETTO, I. Dimensionamento amostral para Avaliação da massa e diâmetro de frutos de nogueira-pecã. Ciência Rural, n.45, v.5, p.794-798, 2015.

CARGNELUTTI FILHO, A.; TOEBE, M.; BURIN, C.; FICK, A. L.; ALVES, B. M.; FACCO, G. Tamanho de amostra para a estimação da média do comprimento, diâmetro e massa de sementes de feijão de porco e mucuna cinza. Ciência Rural, v.42, n.9, p.1541-1544, 2012.

COELHO, A. A.; OLIVEIRA, E. M. S.; RESENDE, E. D.; THIÉBAUT, J. T. L. Dimensionamento amostral para a caracterização da qualidade pós-colheita do maracujá-amarelo. Revista Ceres, v.58, n.1, p.23-28, 2011.

DANTAS, J.L.L.; DANTAS, A. C. L.; LIMA, J. F. Mamoeiro. In: BRUCKNER 
CH. (ed.). Melhoramento de fruteiras tropicais. Viçosa: UFV, 2002, p.309-349.

DIAS, N.L.P.; OLIVEIRA, E. J.; DANTAS, J. L. L. Avaliação de genótipos de mamoeiro com uso de descritores agronômicos e estimação de parâmetros genéticos. Pesquisa Agropecuária Brasileira, v.46, n.11, p.1471-1479, 2011.

EFRON, B. Bootstrap methods: another look at the jackknife. The Annals of Statistics., v.7, n.1, p.1-26, 1979.

FAGUNDES, G.R.; YAMANISHI, O.K. Características físicas e químicas de frutos de mamoeiro do grupo "Solo" comercializados em 4 estabelecimentos de Brasília- DF. Revista Brasileira de Fruticultura, v.23, n.3, p.345-350, 2011.

FAO - Food and Agriculture Organization of the United Nations. Crops. 2018. Disponível em: http://www.fao.org/faostat/en/\#data/QC. Acesso em: 13 de abril de 2020.

FERREIRA, D.F. Estatística básica. 2a .ed. Minas Gerais: UFLA, 2009, 664p.

HELL， L.R.; NASCIMENTO, A. L.; SCHMILDT, O.; MALIKOUSKI, R. G.; FERNANDES, A. A.; BERILLI, S. S.; MORAIS JUNIOR, N. N. M.; QUARTEZANI, W. Z.; AMARAL, J. A. T.; SCHMILDT, E. R. Sample size for evaluation of eggplant and gilo seedlings. African Journal of Agricultural, v.12, n. 34, p.2651-2657, 2017.

IBGE. Instituto Brasileiro de Geografia e Estatística. Produção agrícola municipal: Área plantada ou destinada à colheita, área colhida, quantidade produzida, rendimento médio e valor da produção das lavouras temporárias e permanentes. 2018. Disponível em: http://www.sidra.ibge.gov.br. Acesso em: 13 de abril de 2020.
KRAUSE, W.; STORCK, L.; LUCIO, A. D.; NIED, A. H.; GONÇALVEZ, R. Q. Tamanho ótimo de amostra para avaliação de caracteres de frutos de abacaxizeiro em experimentos com adubação usando parcelas grandes. Revista Brasileira de Fruticultura, v.35, n.1, p.183-190, 2013.

LAW, A.M.; KELTON, W.D. Simulation modeling and analysis. $3^{\mathrm{a}}$ ed. Europe, McGraw-Hill Education. 2000, 759p.

LEVINE, D.M. Estatística: teoria e aplicações usando o Microsoft Excel em português. $6^{\mathrm{a}}$ ed. Rio de Janeiro: LTC Editora. 2012, 832p.

MAPA. Ministério da Agricultura, Pecuária e Abastecimento. 2010. Instrução Normativa 4/2010: Regulamento técnico do mamão. 2010. Disponível em: http://sistemasweb.agricultura.gov.br/sislegi s/action/detalhaAto.method=visualizarAtoP ortalMapa\&chave $=1867613810$. Acesso em: 18 nov. 2018.

NASCIMENTO, A.L.; NASCIMENTO, A. L.; SCHMILDT, O.; SANTOS, K. T. H.; MALIKOUSKI, R. G.; ALEXANDRE, R. S.; CATTANEO, L. F.; AMARAL, J. A. T.; CZEPAK, M. P.; FERREGUETI, G. A.; SCHMILDT, E. R. Evaluation of new papaya hybrids. African Journal of Agricultural, v.13, n.25, p.1283-1290, 2018a.

NASCIMENTO, A.L.; NASCIMENTO, A. L.; SANTOS, K. T. H.; MALIKOUSKI, R. G.; SCHMILDT, O.; ALEXANDRE, R. S.; CZEPAK, M. P.; CATTANEO, L. F.; FERREGUETI, G. A.; AMARAL, J. A. T.; SCHMILDT, E. R. Obtaining and Evaluating New Hybrids of Papaya Tree. Journal of Agricultural Science, v.10, n.7, p.146-155, 2018b.

NAVIDI, W.C. Statistics for engineers and scientists. $3^{\text {a }}$ ed. Boston: McGraw-Hill. 2006, 364p. 
POLETTO, T.; FANTINEL, V. S.; MUNIZ, M. F. B.; DUTRA, A. F. Tamanho de amostra para caracterização de frutos de Carya illinoinensis. Agropecuária Científica no Semiárido, v.14, n.2, p.103107, 2018.

R CORE TEAM. R: a language and environment for statistical computing. Vienna: $\mathrm{R}$ Foundation for Statistical Computing, 2019.

RODOLFO JUNIOR, F.; TORRES, L. B. V.; CAMPOS, V. B.; LIMA, A. R.; OLIVEIRA, A. D.; MOTA, J. K. M. Caracterização físico-química de frutos de mamoeiro comercializados na Empasa de Campina Grande-PB. Revista Brasileira de Produtos Agroindustriais, v.9, n.1, p.5358, 2007.

SCHMILDT, E.R.; ALEXANDRE, R. S.; SIQUEIRA, A. L.; MAYRINCK, L. G.; SCHMILDT, O. Dimensionamento amostral para analisar caracteres físicos e químicos de frutos de maracujá-fedorento. Revista Ceres, v.64, n.2, p.115-121, 2017.

SCHMILDT, E.R.; SCHMILDT, O.; SALINAS, I.; HUESO, J. J.; PINILLOS, V.; CUEVAS, J. Sample size for the evaluation of 'BH-65' papaya fruits under protected cultivation. Revista Brasileira de Fruticultura, v.41, n.2, p.1-9, 2019.

SILVA, W.; BIANCO, A. C.; OLIARI, L. S.; GILES, J. A. D.; SCHMILDT, O.; SCHMILDT, E. R. Dimensionamento amostral para caracterização física e química em frutos de ciriguela. Revista Agro@mbiente On-line, v.10, n.2, p.178182, 2016.

SILVA, C.A.; NASCIMENTO, A. L.; FERREIRA， J. P.; SCHMILDT, O.; MALIKOUSKI, R. G.; ALEXANDRE, R. S.; FERREGUETTI, G. A.; SCHMILDT, E.
R. Genetic diversity among papaya accessions. African Journal of Agricultural, v.12, n.23, p.2041-2048, 2017a.

SILVA, W.; SCHMILDT, E. R.; SCHMILDT, O.; FERREGUETTI, G. A. Dimensionamento amostral para frutos de mamoeiro 'Golden THB' destinados ao mercado nacional e à exportação. Revista Agro@mbiente On-line, v.11, n.2, p.155163, 2017b.

SILVA, M.S.; LEONEL, S.; SOUZA, J. M. A.; MODESTO, J. H.; FERREIRA, R. B.; BOLFARINI, A. C. B. Evaluation of papaya genotypes using agronomic descriptors and estimation of genetic parameters. Bioscience Journal, v.34, n.4, p.943-951, 2018.

SINGH, D. The relative importance of characters affecting genetic divergence. The Indian Journal of Genetics e Plant Breeding, v.41, n.2, p.237-245, 1981.

STORCK, L.; RIBEIRO, N. D.; CARGNELUTTI FILHO, A. Precisão experimental de ensaios de feijão analisada pelo método de Papadakis. Pesquisa Agropecuária Brasileira, v.46, n.8, p.798804, 2011.

TOEBE, M.; BOTH, V.; BRACKMANN, A.; CARGNELUTTI FILHO, A.; THEWES, F. R. Tamanho de amostra para a estimação da média de caracteres de pêssego na colheita e após o armazenamento refrigerado. Ciência Rural, v.42, n.2, p.209-212, 2012.

TOEBE, M.; BOTH, V.; THEWES, F. R.; CARGNELUTTI FILHO, A.; BRACKMANN, A. Tamanho de amostra para a estimação da média de caracteres de maçã. Ciência Rural, v.44, n.5, p.759-767, 2014. 